\title{
Analysis of the Time Complexity of Strassen Algorithm
}

\author{
Xiang Wang \\ School of Information and Electronic Engineering \\ Tianjin Vocational Institute \\ Tianjin, China \\ e-mail: pingfan6699@163.com
}

\begin{abstract}
Algorithms of matrix multiplication are widely used in software engineering field. Application of Strassen algorithm makes a significant contribution to optimize the algorithm . Therefore, thorough study based on time complexity of matrix multiplication algorithm is very important. This paper talks about the time complexity of Strassen algorithm and general algorithm for matrix multiplication, and makes a comparison between the two algorithm routines so as to discuss the advantages and disadvantages of Strassen algorithm. The rational utilization plan of matrix multiplication algorithms is also discussed.
\end{abstract}

Keywords-algorithms for matrix multiplication; time complexity; Strassen algorithm

\section{A GENERAL ALGORITHM FOR MATRIX MULTIPLICATION}

Let $A=\left(a_{i j}\right) m \times n$ be an $m \times n$ matrix, and let $B=\left(b_{i j}\right) n \times p$ be an $n \times p$ matrix. Given an $(m \times p)$ matrix A with $r$ row partitions and s column partitions and $a(p \times n)$ matrix with $s$ row partitions and $\mathrm{t}$ column partitions that are compatible with the partitions of $\mathrm{A}$, the matrix product $\mathrm{C}=\mathrm{A} \times \mathrm{B}$. generally we can explain the matrix multiplication through the following expression, i.e.,

$$
\begin{aligned}
A & =\left(\begin{array}{cccc}
A_{11} & A_{12} & \cdots & A_{1 s} \\
A_{21} & A_{22} & \cdots & A_{2 s} \\
\vdots & \vdots & & \vdots \\
A_{r 1} & A_{r 2} & \cdots & A_{r s}
\end{array}\right) B=\left(\begin{array}{cccc}
B_{11} & B_{12} & \cdots & B_{1 t} \\
B_{21} & B_{22} & \cdots & B_{2 t} \\
\vdots & \vdots & & \vdots \\
B_{s 1} & B_{s 2} & \cdots & B_{s t}
\end{array}\right) . \\
A B & =\left(\begin{array}{cccc}
C_{11} & C_{12} & \cdots & C_{1 t} \\
C_{21} & C_{22} & \cdots & C_{2 t} \\
\vdots & \vdots & & \vdots \\
C_{r 1} & C_{r 2} & \cdots & C_{r t}
\end{array}\right)
\end{aligned}
$$

Thus AB can be formed blockwise, yielding as an $(\mathrm{m} \times \mathrm{p})$ matrix with $\mathrm{r}$ row partitions and $\mathrm{t}$ column partitions. The matrices in your matrix are calculated by multiplying:

$$
C_{i j}=\sum_{\beta=1}^{s} A_{i \beta} B_{\beta j} \text { and } \mathrm{i}=1, \ldots, \mathrm{r} ; \mathrm{j}=1, \ldots, \mathrm{t} .
$$

The function of matrix multiplication is as follows:

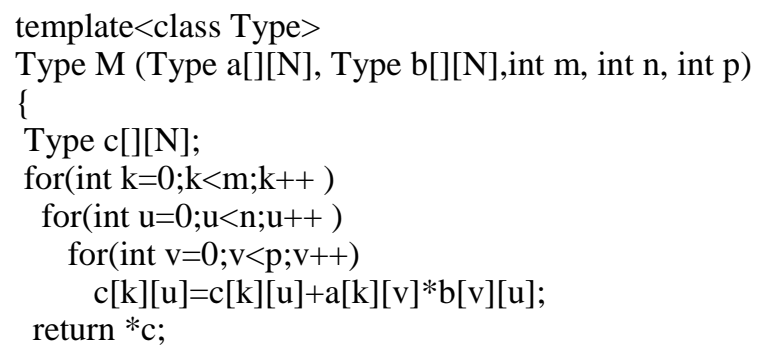

We can obtain the following conclusion very easily: time complexity of a general algorithm for matrix multiplication depends on the rows and columns of matrix A and matrix B., and generally we can explain the time complexity through the following expression, i.e., $\mathrm{O}(\mathrm{m} \times \mathrm{n} \times \mathrm{p})$. If matrix $\mathrm{A}$ and matrix $\mathrm{B}$ are both $\mathrm{n} \times \mathrm{n}$ matrices, $A \times B$ is just a special case of matrix multiplication. And if $n$ is a power of 2, both matrix $A$ and matrix $B$ can be partitioned into $4 \mathrm{n} / 2 \times \mathrm{n} / 2$ blocks. The partitioned matrix can then be written as

$$
\begin{aligned}
& A=\left(\begin{array}{ll}
A_{11} & A_{12} \\
A_{21} & A_{22}
\end{array}\right) \quad B=\left(\begin{array}{ll}
B_{11} & B_{12} \\
B_{21} & B_{22}
\end{array}\right) \\
& A B=\left(\begin{array}{ll}
A_{11} B_{11}+A_{12} B_{21} & A_{11} B_{12}+A_{12} B_{22} \\
A_{21} B_{11}+A_{22} B_{21} & A_{21} B_{12}+A_{22} B_{22}
\end{array}\right)
\end{aligned}
$$

In this case, if we adopt the general algorithm for matrix multiplication, then we need make eight $n / 2 \times n / 2$ Matrix Multiplications and four Matrix additions (or matrix subtractions). Let $T(n)$ be time complexity of matrix multiplication, we can explain the $T(n)$ through the following recursive expression, i.e.,

$T(n)=\left\{\begin{array}{cc}1 & \mathrm{n}=1 \\ 8 \mathrm{~T}\left(\frac{\mathrm{n}}{2}\right)+4\left(\frac{n}{2}\right)^{2} & \mathrm{n}>1\end{array}\right.$

and generally we can explain the time complexity through the following expression, i.e., $\mathrm{O}\left(\mathrm{n}^{3}\right)$. 


\section{ANALYSIS Of Time COMPLEXITy Of THE STRASSEN \\ ALGORITHM}

Strassen algorithm used for matrix multiplication, which is invented by the German mathematician Volker Strassen in 1969, is considered as one of the fastest and best matrix multiplication algorithms. We define 7 new matrices:

$$
\begin{aligned}
& F 1=A_{11}\left(B_{12}-B_{22}\right), \\
& F 2=\left(A_{11}+A_{12}\right) B_{22}, \\
& F 3=\left(A_{21}+A_{22}\right) B_{11}, \\
& F 4=A_{22}\left(B_{21}-B_{11}\right), \\
& F 5=\left(A_{11}+A_{22}\right)\left(B_{11}+B_{22}\right), \\
& F 6=\left(A_{12}-A_{22}\right)\left(B_{21}+B_{22}\right), \\
& F 7=\left(A_{11}-A_{21}\right)\left(B_{11}+B_{12}\right)
\end{aligned}
$$

Thus

$$
A B=\left(\begin{array}{cc}
F_{5}+F_{4}-F_{2}+F_{6} & F_{1}+F_{2} \\
F_{3}+F_{4} & F_{1}+F_{5}-F_{3}-F_{7}
\end{array}\right)
$$

The numbers of $n / 2 \times n / 2$ matrix multiplication may set back to 7 through the Strassen algorithm, when compared with the general algorithm for matrix multiplication. This meantime, there are 18 matrix addition or matrix subtraction. As $\mathrm{n}$ is a power of 2, if let $\mathrm{k}$ be nonnegative integer, then $\mathrm{n}$ is $\mathrm{k}$-th power of 2 . If $\mathrm{k}=0$, then the time complexity of matrix multiplication is 1 . If $k>0$, generally we can explain time complexity of matrix multiplication through the following recursive expression,

$$
\text { i.e., } 7 \mathrm{~T}\left(\frac{\mathrm{n}}{2}\right)+18\left(\frac{\mathrm{n}}{2}\right)^{2}
$$

so we can explain the $\mathrm{T}(\mathrm{n})$ through the following recursive expression,

i.e., $T(n)=\left\{\begin{array}{cc}1 & \mathrm{n}=1 \\ 7 \mathrm{~T}\left(\frac{\mathrm{n}}{2}\right)+18\left(\frac{\mathrm{n}}{2}\right)^{2} & \mathrm{n}>1\end{array}\right.$

If $\mathrm{n}>1$, then

$\mathrm{T}(\mathrm{n})=7 \mathrm{~T}\left(\frac{\mathrm{n}}{2}\right)+18\left(\frac{\mathrm{n}}{2}\right)^{2}$

We come next to the analysis of equation(8). We replace variable $\mathrm{n}$ with $2^{\mathrm{k}}$.

$$
\begin{aligned}
& \mathrm{T}(\mathrm{n})=7 \mathrm{~T}\left(\frac{2^{\mathrm{k}}}{2}\right)+18\left(\frac{2^{\mathrm{k}}}{2}\right)^{2} \\
& =7 \mathrm{~T}\left(2^{\mathrm{k}-1}\right)+18\left(2^{\mathrm{k}-1}\right)^{2} \\
& =7\left(7 \mathrm{~T}\left(2^{\mathrm{k}-2}\right)+18\left(2^{\mathrm{k}-2}\right)^{2}\right)+18\left(2^{\mathrm{k}-1}\right)^{2} \\
& =7^{2} \mathrm{~T}\left(2^{\mathrm{k}-2}\right)+7 \times 18\left(2^{\mathrm{k}-2}\right)^{2}+18\left(2^{\mathrm{k}-1}\right)^{2} \\
& =7^{2}\left(7 \mathrm{~T}\left(2^{\mathrm{k}-3}\right)+18\left(2^{\mathrm{k}-3}\right)^{2}\right) \\
& +7 \times 18\left(2^{\mathrm{k}-2}\right)^{2}+18\left(2^{\mathrm{k}-1}\right)^{2} \\
& =7^{3} \mathrm{~T}\left(2^{\mathrm{k}-3}\right)+7^{2} \times 18\left(2^{\mathrm{k}-3}\right)^{2} \\
& +7 \times 18\left(2^{\mathrm{k}-2}\right)^{2}+18\left(2^{\mathrm{k}-1}\right)^{2} \\
& =\cdots=\sum_{i=0}^{k-1} 7^{i} \times 18\left(2^{\mathrm{k}-\mathrm{i}-1}\right)^{2} \\
& =18\left(2^{\mathrm{k}}\right)^{2} \sum_{i=0}^{k-1} \frac{7^{i}}{\left(2^{\mathrm{i}+1}\right)^{2}} \\
& =\frac{9}{2}\left(2^{\mathrm{k}}\right)^{2} \sum_{i=0}^{k-1}\left(\frac{7}{4}\right)^{i} \\
& =\frac{9}{2} \times\left(2^{\mathrm{k}}\right)^{2} \times \frac{1-\left(\frac{7}{4}\right)^{k}}{1-\frac{7}{4}} \\
& =6 \times 7^{k}-6 \times 4^{k}
\end{aligned}
$$

Since

$$
\begin{aligned}
& 7^{\mathrm{k}}=7^{\log _{2} n} \\
& =n^{\log _{n} 7^{\log _{2} n}} \\
& =n^{\log _{2} n \log _{n} 7} \\
& =n^{\log _{2} n \frac{\log _{2} 7}{\log _{2} n}} \\
& =n^{\log _{2} 7} \\
& \approx n^{2.807355} \\
& \approx n^{2.81}
\end{aligned}
$$

and 


$$
\begin{aligned}
& 4^{k}=2^{2 k}=2^{2 \log _{2} n} \\
& =2^{\log _{2} n^{2}}=n^{2}
\end{aligned}
$$

Then, we have

$$
T(n) \approx\left\{\begin{array}{cc}
1 & \mathrm{n}=1 \\
6 n^{2.81}-6 n^{2} & \mathrm{n}>1
\end{array}\right.
$$

This meantime, we can explain the time complexity through the following expression, i.e., $\mathrm{O}\left(\mathrm{n}^{2.81}\right)$. It is evident that the Strassen algorithm was more efficient than the general algorithm for matrix multiplication. In fact, if $n$ is not a power of 2, the matrix can be embedded into another matrix (let the matrix be matrix E) whose number of dimension is a power of 2 . In matrix multiplication, if the dimension of matrix $\mathrm{E}$ take minimum value within the design requirement above is achieved, the dimension will increase twofold at most. Thus, the time complexity of Strassen algorithm can still be described as $\mathrm{O}\left(\mathrm{n}^{2.81}\right)$.

\section{VARIATION TRENDS Of TIME COMPLEXITY OF THE STRASSEN ALGORITHM}

For the general algorithm for matrix multiplication, let $\mathrm{n}$ be a power of 2 . If let $\mathrm{k}$ be nonnegative integer, then $\mathrm{n}$ is $\mathrm{k}$-th power of 2 , i.e., $n=2^{k}$. When $n>1$, we can deeply analyze time complexity of the general algorithm for matrix multiplication through the following recursive expression,

$$
\mathrm{T}(\mathrm{n})=8 \mathrm{~T}\left(\frac{\mathrm{n}}{2}\right)+4\left(\frac{n}{2}\right)^{2}
$$

We come next to the analysis of equation(10). We replace variable $\mathrm{n}$ with $2^{\mathrm{k}}$.

$$
\begin{aligned}
& \mathrm{T}(\mathrm{n})=8 T\left(\frac{2^{k}}{2}\right)+4\left(\frac{2^{k}}{2}\right)^{2} \\
& =8 T\left(2^{k-1}\right)+4\left(2^{k-1}\right)^{2} \\
& =\cdots=\sum_{i=0}^{k-1} 8^{i} \times 4\left(2^{\mathrm{k}-\mathrm{i}-1}\right)^{2} \\
& =4\left(2^{\mathrm{k}}\right)^{2} \sum_{i=0}^{k-1} \frac{8^{i}}{\left(2^{\mathrm{i}+1}\right)^{2}} \\
& =\left(2^{\mathrm{k}}\right)^{2} \sum_{i=0}^{k-1} 2^{i}
\end{aligned}
$$

$$
\begin{aligned}
& =\left(2^{\mathrm{k}}\right)^{2} \times \frac{1-2^{k}}{1-2} \\
& =2^{3 k}-2^{2 k} \\
& =2^{3 \log _{2} n}-2^{2 \log _{2} n} \\
& =n^{3}-n^{2}
\end{aligned}
$$

Thus, we have

$$
T(n)=\left\{\begin{array}{cc}
1 & \mathrm{n}=1 \\
n^{3}-n^{2} & \mathrm{n}>1
\end{array}\right.
$$

Let us begin to consider the behavior of a new function:

$$
\begin{aligned}
& K(n)=\left(n^{3}-n^{2}\right)-\left(n^{2.81}-6 n^{2}\right) \\
& \text { i.e., } K(n)=n^{3}-n^{2.81}+5 n^{2}
\end{aligned}
$$

We can easily get the first order derivative and second order derivative of the function, such that

$$
\begin{aligned}
& K^{\prime}(n)=3 n^{2}-2.81 n^{1.81}+10 n \\
& K^{\prime \prime}(n)=6 n-5.0861 n^{0.81}+10
\end{aligned}
$$

We can obtain that if $\mathrm{n}>1$, then $K^{\prime}(n)>0$ and $K^{\prime \prime}(n)>0$. This implies that Strassen algorithm is always better than the general algorithm for matrix multiplication in terms of time complexity. And the advantage of the Strassen algorithm will became more obvious with the increase of dimension of matrix. Strassen algorithm adopts the method of recursive algorithms, which is helpful to the implementation and analysis of the algorithm. However, the method make the algorithm require a great number of dynamic two dimensional arrays so as to assign memory space. which enhance the time complexity and space complexity when the dimension of matrix is smaller. However, the general algorithm for matrix multiplication is no problem on this aspect. Tests show when the dimension of matrix is less than 500, this situation will get even worse. In order to solve this problem, we can specify a numeric value (such as 500) for algorithm routine, and we will adopt Strassen algorithm or the general algorithm for matrix multiplication based on the comparisons between the dimension and the numeric value.

The function of Strassen algorithm is as follows: 
